\title{
High prevalence and clonal dissemination of OXA-72-producing Acinetobacter baumannii in a Chinese hospital: a cross sectional study
}

Yong Chen ${ }^{1+}$, Yuying Yang ${ }^{2,3+}$, Lin Liu ${ }^{1,4+}$, Guangbin Qiu ${ }^{5}$, Xuelin Han ${ }^{1}$, Shuguang Tian ${ }^{1}$, Jingya Zhao ${ }^{1}$, Fangyan Chen ${ }^{1}$, Hajo Grundmann ${ }^{6,7}$, Haifeng Li ${ }^{8^{*}}$, Jinke Sun ${ }^{2,3,9^{*}}$ and Li Han ${ }^{1 *}$

\begin{abstract}
Background: Carbapenem resistance in Acinetobacter baumannii in China was mainly mediated by OXA-23-like carbapenemases, while OXA-24/40-like carbapenemases were rarely identified. OXA-72 is one variant of OXA-24/40-like carbapenemases. This study aimed to demonstrate the epidemiology and characterizations of OXA-72-producing A. baumannii in a Chinese hospital.

Methods: A total of 107 clinical A. calcoaceticus-A. baumannii (Acb) complex isolates were collected in a Chinese hospital during between 2014 and 2016. These isolates were identified using Vitek 2 system and gyrB multiplex PCR. Vitek 2 system was used for antibiotic susceptibility testing. Genes encoding for major classes of carbapenemases were investigated by PCR. Rep-PCR was used for genotyping of all the A. baumannii isolates. The risk factors for carriage of OXA-72-producing or OXA-23-producing A. baumannii were analyzed through univariate and multivariate logistic regression.

Results: Of the 107 Acb isolates collected, 101 isolates (94.4\%) and 6 isolates (5.6\%) were identified as A. baumannii and A. pittii, respectively. 78 A. baumannii isolates (77.2\%) were carbapenem resistant and mainly cultured from intensive care unit (ICU). bla $a_{\mathrm{OXA}-72}$ and bla $a_{\mathrm{OXA}-23}$ genes were identified in $45(57.7 \%)$ and 33(42.3\%) carbapenem-resistant A. baumannii (CRAB), respectively. Multivariate risk factor analyses showed that prior carbapenem usage and nasogastric intubation were significantly associated with carriage of OXA-72-producing A. baumannii or OXA-23-producing A. baumannii. Rep-PCR analysis showed that 9 and 22 Rep-PCR types were assigned to 78 CRAB isolates and 23 carbapenem-susceptible $A$. baumannii (CSAB) isolates, respectively. A higher diverstiy of Rep-PCR patterns was observed among OXA-72-producing A. baumannii isolates than OXA-23-producing A. baumannii isolates, but all of them belonged to the same clone complex. MLST analysis suggested that the OXA-72 isolates from this study correspond to CC92/CC2 clone complex.

(Continued on next page)
\end{abstract}

\footnotetext{
* Correspondence: 185117683@qq.com; sunjinke202@sina.com; hanlicdc@163.com

†Yong Chen, Yuying Yang and Lin Liu contributed equally to this work. ${ }^{8}$ Department of Hospital Infection Control, The 202nd Hospital of PLA, Shenyang 110003, China

${ }^{2}$ School of Public Health, Shenyang Medical College, Shenyang, China

'Department of Hospital Infection Control, Chinese PLA Institute for Disease

Control and Prevention, 20\# Dongda Str, Beijing 100071, China

Full list of author information is available at the end of the article
}

(c) The Author(s). 2018 Open Access This article is distributed under the terms of the Creative Commons Attribution 4.0 International License (http://creativecommons.org/licenses/by/4.0/), which permits unrestricted use, distribution, and reproduction in any medium, provided you give appropriate credit to the original author(s) and the source, provide a link to the Creative Commons license, and indicate if changes were made. The Creative Commons Public Domain Dedication waiver (http://creativecommons.org/publicdomain/zero/1.0/) applies to the data made available in this article, unless otherwise stated. 
(Continued from previous page)

Conclusions: This study demonstrates high prevalence and potential clonal spread of closely related genotypes of

OXA-72-producing A. baumannii within a Chinese hospital. Continuous surveillance is necessary to monitor the

dissemination of these strains in other healthcare settings to guide infection control policies in order to curb the

spread of this bacterium.

Keywords: Acinetobacter baumannii, OXA carbapenemases, Risk factor, Molecular typing, Clone dissemination

\section{Background}

Acinetobacter is a gram-negative coccobacillus that has rapidly emerged as one of the most common nosocomial pathogens worldwide [1]. There are currently at least 31 described Acinetobacter genomic species [2], of which A. calcoaceticus, A. baumannii, A. pittii, and A. nosocomialis are very closely related, and difficult to distinguish from each other by phenotypic properties [3]. A. calcoaceticus-A. baumannii (Acb) complex has therefore been proposed to refer to these species [2]. Nevertheless, A. baumannii is the most clinically relevant and is notorious for its ability to accumulate diverse mechanisms of resistance $[4,5]$.

The carbapenem class of antibiotics is considered as the last-resort choice when treating Acinetobacter infections. However, an increasing prevalence of carbapenem resistance has been observed in clinical $A$. baumannii isolates from many parts of the world. Carbapenem-associated multiclass resistance among 55,330 U.S. A. baumannii isolates from The Surveillance Network database has increased from $20.6 \%$ in 2002 to $49.2 \%$ in 2008 [6]. In China, the resistance rate of clinical $A$. baumannii to carbapenem gradually increased from $<10 \%$ in 2000 to $>60 \%$ at present $[7,8]$. The major mechanism of carbapenem resistance is production of OXA $\beta$-lactamases, which are clustered in three major groups, OXA-23-like, OXA-24/ 40-like, and OXA-58-like [9-11].

OXA-24/40 $\beta$-lactamase was first identified in $A$. baumannii from Spain in 1997 [12]. After that, A further 6 enzyme variants have since been discovered, including OXA-72 [11]. Unlike OXA-23-like, OXA-24/40 $\beta$-lactamases were less commonly identified in carbapenem resistant Acinetobacter spp. isolates [13]. A large surveillance of OXA-type $\beta$-lactamase gene clusters for a total of 2880 Acinetobacter spp. isolates collected from 23 Chinese provinces found that bla $a_{\text {OXA-23-like }}$ and $b l a_{\text {OXA-24/40-like genes were identified in } 1316 \text { isolates }}$ (45.7\%) and 11 isolates (0.4\%), respectively [14]. Therefore, OXA-24/40-like $\beta$-lactamases were only responsible for a small number of carbapenem-resistance isolates in China, their dissemination and epidemiology in healthcare settings deserves further surveillance and investigation.

This study aimed to demonstrate the occurrence, clinical manifestation and genotypic characterizations of OXA-72-producing A. baumannii in a Chinese hospital.

\section{Methods}

Study settings and isolates information

A total of 107 clinical Acb complex isolates from 107 patients were collected at a tertiary-care comprehensive hospital in northeastern China with 1800 beds, from Oct 2014 to Oct 2016. These isolates were recovered from various specimens including sputum, blood, urine, pleural fluid, secretions and throat swab sample. For each patient, only a single colony of the first isolate was selected for subsequent analysis. All the clinical isolates were stored at $-80{ }^{\circ} \mathrm{C}$ until use. Data for each isolate and each patient were obtained through review of microbiology lab results and medical records. Risk factor data, including ICU stay, presence of invasive procedures and antibiotic treatment, referred to those which were present before the isolation of the index Acinetobacter strains. All data were anonymously collected and interpreted.

\section{Strain identification and in vitro antibiotic susceptibility testing}

Identification of Acb complex isolates was initially performed using automated identification systems the VITEK 2 compact system (BioMérieux, Craponne, France). Further identification of the Acb complex to the species level was performed by gyrB multiplex PCR $[15,16]$.

In vitro susceptibilities to ampicillin-sulbactam, piperacillintazobactam, ceftazidime, cefepime, meropenem, imipenem, gentamicin, amikacin, ciprofloxacin, levofloxacin, colistin, minocyline were determined by the VITEK 2 compact system (BioMérieux, Craponne, France). Escherichia coli (ATCC 25922) and Pseudomonas aeruginosa (ATCC 27853) were used as quality control strains. Results were interpreted according to the Clinical and Laboratory Standards Institute (CLSI, 2016) guidelines.

\section{Detection of carbapenemases genes}

One white loop $(1 \mu \mathrm{l})$ of $24 \mathrm{~h}$ plate culture of Acinetobacter bacteria was resuspended in $200 \mu \mathrm{l}$ of sterilized and DNA free water. The bacterial suspensions were then heated for $10 \mathrm{~min}$ at $96{ }^{\circ} \mathrm{C}$ and centrifuged $5 \mathrm{~min}$ at $13000 \mathrm{rpm}$. The supernatant was used as the genomic DNA for the following molecular experiments.

Genes encoding for major classes of A, B, and D carbapenemases for all the Acb isolates were investigated by PCR. The presence of $b l a_{\text {OXA-23-like }}, b l a_{\text {OXA-24-like, }} b l a_{\text {OXA-51-like, }}$ 
and $b l a_{\text {OXA-58-like }}$ genes were detected through multiplex PCR assay [17]. Metallo- $\beta$-lactamase encoding genes, $b l a_{\mathrm{NDM}-\text {-like, }} b l a_{\mathrm{IMP}-\text { like }}$ and $b l a_{\mathrm{VIP}-\text { like, }}$ were detected with PCR conditions and primers as previously described [18]. The bla $a_{\text {OXA-24-like }}$ variant was further identified through PCR and DNA sequencing as described [19].

\section{Molecular typing}

Rep-PCR was used for genotyping of all the A. baumannii isolates, using the primer pair REP 1(5'-IIIGCGC CGICAGGC-3') and REP 2(5'-ACGTCTTATCAGGC CTAC-3') [20]. The conditions for PCR and the gel electrophoresis were the same as previously described [21]. Rep-PCR results of each isolate was compared to all of the other isolates in a pairwise manner, isolates with identical band patterns were considered to be of identical Rep-PCR types. A minimum spanning tree was created to show the differences between patterns through BioNumerics 6.6 software (Applied Maths, Kortrijk, Belgium). The spanning tree was limited to those patterns that differ by a single band, two Rep-PCR types that differ by two or more bands were connected. Nine bla $a_{\text {OXA-24-like }}$ gene positive representative isolates from each different Rep-PCR type were randomly chosen for multilocus sequence typing (MLST) analysis according to 'Pasteur' scheme [22].

\section{A review of literature}

Currently, there were two MLST schemes available for Acinetobacter. In order to differentiate between the two schemes, STs and CCs were designated as $\mathrm{ST}^{\mathrm{B}} / \mathrm{CC}^{\mathrm{B}}$ for the Bartual scheme and $\mathrm{ST}^{\mathrm{P}} / \mathrm{CC}^{\mathrm{P}}$ for the Pasteur scheme. A search of previous published papers giving the MLST results of OXA-24/40-like producing Acinetobacter spp. was conducted to illustrate the population structure of OXA-24/40 strains from different countries. The MLST data from 'Pasteur' scheme and 'Bartual' scheme were separately analyzed by Bionumerics 6.6 and presented as a minimum spanning tree for categorical data with default settings.

\section{Statistical analysis}

Statistical analyses were performed using SPSS 19.0 (IBM, Armonk, NY, USA). The comparisons of patients' characterics were conducted by chi-square test or Mann-Whitney $U$ test. The risk factors for carriage of $b a_{\text {OXA-72-positive or }} b l a_{\text {OXA-23-like-positive A. bauman- }}$ nii were analyzed through univariate and multivariate logistic regression. In multivariate logistic regression, ICU stay and length of ICU stay were not included as they were highly associated with many other predisposing factors, including invasive procedures and antibiotic use. The index of diversity and the 95\% confidence intervals (CIs) were calculated as described previously [23]. $P$ values $<0.05$ are considered statistically significant.

\section{Results}

Of the 107 Acinetobacter isolates, 101 isolates (94.4\%) and 6 isolates (5.6\%) were identified as A. baumannii and $A$. pittii, respectively. Five $A$. pittii isolates were susceptible to all the antibiotics tested and one isolate exhibited an intermediate resistance phenotype to minocycline. Among the $101 \mathrm{~A}$. baumannii isolates, 78 isolates (77.2\%) were resistant to carbapenems (meropenem or imipenem). The rates of resistance to piperacilin/tazobactam, ampicillin/sulbactam, amikacin, gentamicin, ceftazidime, ciprofloxacin and levofloxacin in A. baumannii were all above $60 \%$, as most of the carbapenem-resistance isolates exhibited a multidrug resistance phenotype. Four A. baumannii isolates were resistant to polymyxin.

The 107 Acinetobacter isolates were cultured from 71 male and 46 female patients. Fifty-one isolates were cultured from patients in ICU and all these isolates were carbapenem-resistant. The detection of carbapenemase genes showed that $33(42.3 \%)$ of the 78 carbapenem-resistant $A$. baumannii (CRAB) were positive for the $b l a_{\text {OXA-23-like }}$ gene, while the other 45 isolates (57.7\%) were positive for the $b l a_{\mathrm{OXA}-24 / 40 \text {-like }}$ gene. All these isolates harboured bla OXA-51-like gene. DNA sequencing showed that all the bla $a_{\text {OXA-24/40-like ampli- }}$ cons belonged to bla $a_{\text {OXA-72 }}$ (GenBank accession number

Table 1 Characteristics of 101 patients colonized or infected with Acinetobacter baumannii in a Chinese hospital

\begin{tabular}{|c|c|c|c|c|}
\hline \multirow[t]{2}{*}{ Characteristics } & \multicolumn{2}{|c|}{$\begin{array}{l}\text { No. of patients colonized or infected with } \\
\text { A. baumannii }\end{array}$} & \multirow[t]{2}{*}{$x^{2}$} & \multirow[t]{2}{*}{$P$ values } \\
\hline & $\begin{array}{l}\text { Carbapenem-resistant } \\
(n=78)\end{array}$ & $\begin{array}{l}\text { Carbapenem-susceptible } \\
(n=23)\end{array}$ & & \\
\hline Age, median years(range) & $77(21,94)$ & $76(2,96)$ & - & 0.463 \\
\hline Male Gender, n(\%) & $50(64.1)$ & 19(82.6) & 2.81 & 0.094 \\
\hline Length of hospital stay, median days (range) & $11(1,3650)$ & $8(1,1400)$ & - & 0.786 \\
\hline ICU stay, n (\%) & $49(62.8)$ & $1(4.3)$ & 24.30 & $<0.001$ \\
\hline Associated with an infection, $\mathrm{n}(\%)$ & $67(85.9)$ & 17(73.9) & 1.07 & 0.302 \\
\hline All-cause mortality of patients 14 days after isolation of A. baumannii, $\mathrm{n}(\%)$ & $16(20.5)$ & $4(17.4)$ & 0.001 & 0.974 \\
\hline
\end{tabular}


Table 2 Univariate logistic analysis of risk factors for carriage of OXA-72-producing Acinetobacter baumannii or OXA-23-producing A. baumannii

\begin{tabular}{|c|c|c|c|c|c|c|c|}
\hline Characteristics & $\begin{array}{l}\text { Control cases }^{a} \\
(n=23)\end{array}$ & $\begin{array}{l}\text { OXA-72 cases } \\
(n=45)\end{array}$ & OR(Cl 95\%) & $P$ value & $\begin{array}{l}\text { OXA-23 cases } \\
(n=33)\end{array}$ & OR(Cl 95\%) & $P$ value \\
\hline Age, median(range) & $76(2,96)$ & $78(28,94)$ & $1.02(1.00,1.05)$ & 0.081 & $78(21,90)$ & $1.00(0.99,1.03)$ & 0.460 \\
\hline Male gender, n(\%) & $22(75.9)$ & $27(60.0)$ & $0.32(0.09,1.08)$ & 0.067 & $23(69.7)$ & $0.48(0.13,1.79)$ & 0.484 \\
\hline ICU stay, n (\%) & $1(4.3)$ & $27(60.0)$ & $33.00(4.08,267.04)$ & 0.001 & $22(66.7)$ & $44.00(5.23,370.52)$ & $<0.001$ \\
\hline $\begin{array}{l}\text { Length of stay in the ICU (days), } \\
\text { median(range) }\end{array}$ & $0(0,2)$ & $5(0,100)$ & $2.29(1.20,4.37)$ & 0.012 & $3(0,100)$ & $2.29(1.03,5.08)$ & 0.042 \\
\hline \multicolumn{8}{|l|}{ Predisposing factors } \\
\hline Urinary catheter & $9(39.1)$ & $27(60.0)$ & $2.33(0.84,6.52)$ & 0.106 & $23(69.7)$ & $3.58(1.17,10.96)$ & 0.026 \\
\hline Mechanical ventilation & $2(8.7)$ & 18(40.0) & $7.00(1.46,33.59)$ & 0.015 & $14(42.4)$ & $7.74(1.55,38.56)$ & 0.013 \\
\hline Central venous catheter & $5(21.7)$ & 14(31.1) & $1.63(0.50,5.26)$ & 0.417 & $17(51.5)$ & $3.83(1.15,12.74)$ & 0.029 \\
\hline Tracheostomy & $1(4.3)$ & $10(22.2)$ & $6.29(0.75,52.56)$ & 0.090 & $11(33.3)$ & $11.00(1.31,92.63)$ & 0.027 \\
\hline Transfusion & $1(4.3)$ & $5(11.1)$ & $2.75(0.30,25.05)$ & 0.369 & $7(21.2)$ & $5.92(0.68,51.92)$ & 0.108 \\
\hline Nasogastric intubation & $4(17.4)$ & $28(62.2)$ & $7.82(2.27,26.91)$ & 0.001 & 19(57.6) & $6.45(1.79,23.19)$ & 0.004 \\
\hline Cephalosporin & $12(52.2)$ & 15(33.3) & $0.46(0.16,1.27)$ & 0.136 & $12(36.4)$ & $0.53(0.17,1.55)$ & 0.242 \\
\hline $\begin{array}{l}\beta \text {-lactam } / \beta \text {-lactamase inhibitor } \\
\text { combinations }\end{array}$ & $12(52.2)$ & $24(53.3)$ & $2.61(0.90,7.57)$ & 0.077 & $12(36.4)$ & $1.31(0.42,4.07)$ & 0.645 \\
\hline Quinolone & $3(13.0)$ & 19(42.2) & $4.87(1.26,18.79)$ & 0.022 & 10(30.3) & $2.90(0.70,12.02)$ & 0.143 \\
\hline Carbapenem & $4(17.4)$ & $21(46.7)$ & $4.16(1.22,14.18)$ & 0.023 & 20(60.6) & $7.31(2.02,26.40)$ & 0.002 \\
\hline
\end{tabular}

aControl cases: 23 patients colonized or infected with carbapenem-susceptible Acinetobacter baumannii

ICU intensive care unit

MF781069). The $b l a_{\text {OXA-58-like }}$ gene was detected in one carbapenem-susceptible A. baumannii (CSAB) isolate, the MICs for imipenem and meropenem were $0.5 \mu \mathrm{g} / \mathrm{ml}$ and $0.25 \mu \mathrm{g} / \mathrm{ml}$, respectively. None of these Acinetobacter isolates were positive for $b l a_{\mathrm{IMP}-\mathrm{like}}, b l a_{\mathrm{VIM} \text {-like }}$ or $b l a_{\mathrm{NDM} \text {-like }}$ genes.

Eighty-four (83.2\%) of $101 \mathrm{~A}$. baumannii isolates were associated with an infection (primarily low respiratory tract infection) and antibiotic treatment, while the other 27 isolates were just colonized. The characterics of 101 patients colonized or infected with A. baumannii were shown in Table 1. There were no significant differences over age and gender distribution among patients colonized or infected with CRAB and CSAB strains. More than $60 \%$ of the CRAB patients stayed at ICU at the time of bacteria isolation, while only one CSAB patient stayed at ICU.

The results of univariate logistic analysis showed that there were many common risk factors for carriage of OXA-72-producing A. baumannii or OXA-23-producing
A. baumannii, such as ICU stay, mechanical ventilation, nasogastric intubation and carbapenem treatment (Table 2). In multivarite analysis, prior carbapenem usage and nasogastric intubation were significantly associated with carriage of OXA-72-producing A. baumannii or OXA-23-producing A. baumannii. An additional risk factor, urinary catheter, was also significantly associated with carriage of bla $a_{\mathrm{OXA}-23-\text { like-positive } A \text {. baumannii }}$ (Table 3).

The results of Rep-PCR patterns and corresponding strain information for the $101 \mathrm{~A}$. baumannii were shown in Table 4. In total, 9 Rep-PCR types were assigned to 78 CRAB isolates, while 22 Rep-PCR types were assigned to $23 \mathrm{CSAB}$ isolates. The index of diversity (DI) for CRAB was 0.750 (95\% CI: 0.671-0.829), which was significantly lower than for CSAB (DI =0.996, 95\% CI: 0.986-1.006). Twenty-six and 7 OXA-23-producing A. baumannii isolates were identified as Rep-PCR type 1 and 2, respectively, while 45 OXA-72-producing A. baumannii isolates

Table 3 Multivariate logistic analysis of risk factors for carriage of OXA-72-producing Acinetobacter baumannii or OXA-23-producing A. baumannii

\begin{tabular}{lllll}
\hline Characteristics & OXA-72 cases & & OXA-23 cases \\
\cline { 2 - 3 } & OR(Cl 95\%) & $P$ value & OR(Cl 95\%) & \\
\hline Urinary catheter & - & - & $4.94(1.61-21.07)$ & \\
Nasogastric intubation & $7.65(2.14,27.36)$ & 0.002 & $7.95(1.79-35.34)$ & 0.031 \\
Carbapenem usage & $4.02(1.07,15.06)$ & 0.039 & $10.05(2.21-45.58)$ & 0.006 \\
\hline
\end{tabular}


Table 4 The Rep-PCR type, MLST type, presence of carbapenemase genes and antimicrobial resistance profile of 101 Acinetobacter baumannii isolates

\begin{tabular}{|c|c|c|c|c|c|c|c|c|c|c|c|c|c|c|c|}
\hline \multirow{2}{*}{$\begin{array}{l}\text { Isolate } \\
\text { ID }\end{array}$} & \multirow{2}{*}{$\begin{array}{l}\text { Rep-PCR } \\
\text { type }\end{array}$} & \multirow{2}{*}{$\begin{array}{l}\text { MLST } \\
\text { type }\end{array}$} & \multirow{2}{*}{$\begin{array}{l}\text { Carbapenemase } \\
\text { genes }\end{array}$} & \multicolumn{12}{|c|}{ Antimicrobial resistance profile } \\
\hline & & & & $\overline{I M P}$ & MEM & CAZ & FEP & AMK & GEN & CIP & LVX & TZP & SAM & $\mathrm{MH}$ & $\mathrm{COL}$ \\
\hline N1312 & 1 & NA & $b / a_{\text {OXA-23 }}$ & $\mathrm{R}$ & $\mathrm{R}$ & $\mathrm{R}$ & $\mathrm{R}$ & $\mathrm{R}$ & $\mathrm{R}$ & $\mathrm{R}$ & $\mathrm{R}$ & $\mathrm{R}$ & $\mathrm{R}$ & $\mathrm{S}$ & S \\
\hline N1314 & 1 & NA & $b / a_{\text {OXA-23 }}$ & $\mathrm{R}$ & $\mathrm{R}$ & $\mathrm{R}$ & । & $\mathrm{R}$ & $\mathrm{R}$ & $\mathrm{R}$ & $\mathrm{R}$ & $\mathrm{R}$ & $\mathrm{R}$ & $\mathrm{S}$ & S \\
\hline N1316 & 1 & NA & $b / a_{\mathrm{OXA}-23}$ & $\mathrm{R}$ & $\mathrm{R}$ & $\mathrm{R}$ & $\mathrm{R}$ & $\mathrm{R}$ & $\mathrm{R}$ & $\mathrm{R}$ & $\mathrm{R}$ & $\mathrm{R}$ & $\mathrm{R}$ & $\mathrm{S}$ & S \\
\hline N1318 & 1 & NA & bla OXA-23 & $\mathrm{R}$ & $\mathrm{R}$ & $\mathrm{R}$ & । & $S$ & $\mathrm{R}$ & $\mathrm{R}$ & $\mathrm{R}$ & $\mathrm{R}$ & I & $S$ & S \\
\hline N1352 & 1 & NA & $b / a_{\mathrm{OXA}-23}$ & $\mathrm{R}$ & $\mathrm{R}$ & $\mathrm{R}$ & $\mathrm{R}$ & $\mathrm{R}$ & $\mathrm{R}$ & $\mathrm{R}$ & I & $\mathrm{R}$ & $\mathrm{R}$ & $S$ & S \\
\hline N1359 & 1 & NA & $b / a_{\mathrm{OXA}-23}$ & $\mathrm{R}$ & $\mathrm{R}$ & $\mathrm{R}$ & $\mathrm{R}$ & $\mathrm{R}$ & $\mathrm{R}$ & $\mathrm{R}$ & 1 & R & $\mathrm{R}$ & $\mathrm{S}$ & S \\
\hline N1362 & 1 & NA & $b / a_{\mathrm{OXA}-23}$ & $\mathrm{R}$ & $\mathrm{R}$ & $\mathrm{R}$ & $\mathrm{R}$ & $\mathrm{R}$ & $\mathrm{R}$ & $\mathrm{R}$ & $\mathrm{R}$ & $\mathrm{R}$ & $\mathrm{R}$ & $S$ & S \\
\hline N1369 & 1 & NA & $b / a_{\mathrm{OXA}-23}$ & $\mathrm{R}$ & $\mathrm{R}$ & $\mathrm{R}$ & $\mathrm{R}$ & $\mathrm{R}$ & 1 & $\mathrm{R}$ & I & $\mathrm{R}$ & $\mathrm{R}$ & $S$ & S \\
\hline N1371 & 1 & NA & bla OXA-23 & $\mathrm{R}$ & $\mathrm{R}$ & $\mathrm{R}$ & $\mathrm{R}$ & $\mathrm{R}$ & $\mathrm{R}$ & $\mathrm{R}$ & I & $\mathrm{R}$ & $\mathrm{R}$ & $S$ & S \\
\hline N1383 & 1 & NA & $b / a_{\text {OXA-23 }}$ & $\mathrm{R}$ & $\mathrm{R}$ & $\mathrm{R}$ & $\mathrm{R}$ & $\mathrm{R}$ & $\mathrm{R}$ & $\mathrm{R}$ & $\mathrm{R}$ & $\mathrm{R}$ & $\mathrm{R}$ & $S$ & S \\
\hline N1384 & 1 & NA & $b / a_{\mathrm{OXA}-23}$ & $\mathrm{R}$ & $\mathrm{R}$ & $\mathrm{R}$ & $\mathrm{R}$ & $\mathrm{R}$ & $\mathrm{R}$ & $\mathrm{R}$ & $\mathrm{R}$ & $\mathrm{R}$ & $\mathrm{R}$ & $S$ & S \\
\hline N1386 & 1 & NA & $b / a_{\mathrm{OXA}-23}$ & $\mathrm{R}$ & $\mathrm{R}$ & $\mathrm{R}$ & $\mathrm{R}$ & $\mathrm{R}$ & $\mathrm{R}$ & $\mathrm{R}$ & I & $\mathrm{R}$ & $\mathrm{R}$ & $S$ & $R$ \\
\hline N1395 & 1 & NA & $b / a_{\text {OXA-23 }}$ & $\mathrm{R}$ & $\mathrm{R}$ & $\mathrm{R}$ & $\mathrm{R}$ & $\mathrm{R}$ & $\mathrm{R}$ & $\mathrm{R}$ & $\mathrm{R}$ & $\mathrm{R}$ & $\mathrm{R}$ & $S$ & S \\
\hline N1396 & 1 & NA & $b / a_{\mathrm{OXA}-23}$ & $\mathrm{R}$ & $\mathrm{R}$ & $\mathrm{R}$ & $\mathrm{R}$ & $\mathrm{R}$ & $\mathrm{R}$ & $\mathrm{R}$ & $\mathrm{R}$ & $\mathrm{R}$ & $\mathrm{R}$ & S & S \\
\hline N1398 & 1 & NA & $b / a_{\text {OXA-23 }}$ & I & $\mathrm{R}$ & $\mathrm{R}$ & $\mathrm{R}$ & $\mathrm{R}$ & $\mathrm{R}$ & $\mathrm{R}$ & $\mathrm{R}$ & $R$ & I & $S$ & S \\
\hline N1401 & 1 & NA & $b / a_{\mathrm{OXA}-23}$ & $\mathrm{R}$ & $\mathrm{R}$ & $\mathrm{R}$ & $\mathrm{R}$ & $\mathrm{R}$ & $\mathrm{R}$ & $\mathrm{R}$ & $\mathrm{R}$ & $\mathrm{R}$ & I & S & S \\
\hline N1407 & 1 & NA & bla OXA-23 & $\mathrm{R}$ & $\mathrm{R}$ & $\mathrm{R}$ & $\mathrm{R}$ & $\mathrm{R}$ & $\mathrm{R}$ & $\mathrm{R}$ & $\mathrm{R}$ & $\mathrm{R}$ & $\mathrm{R}$ & $S$ & S \\
\hline N741 & 1 & NA & $b l a_{\mathrm{OXA}-23}$ & $\mathrm{R}$ & $\mathrm{R}$ & $\mathrm{R}$ & $\mathrm{R}$ & $\mathrm{R}$ & $\mathrm{R}$ & $\mathrm{R}$ & $\mathrm{R}$ & R & $\mathrm{R}$ & S & S \\
\hline N747 & 1 & NA & $b / a_{\mathrm{OXA}-23}$ & $\mathrm{R}$ & $\mathrm{R}$ & $\mathrm{R}$ & I & $\mathrm{R}$ & $\mathrm{R}$ & $\mathrm{R}$ & $\mathrm{R}$ & $\mathrm{R}$ & $\mathrm{S}$ & I & S \\
\hline N748 & 1 & NA & $b / a_{\mathrm{OXA}-23}$ & $\mathrm{R}$ & $\mathrm{R}$ & $\mathrm{R}$ & $\mathrm{R}$ & $\mathrm{R}$ & $\mathrm{R}$ & $\mathrm{R}$ & $\mathrm{R}$ & S & $\mathrm{R}$ & I & S \\
\hline N749 & 1 & NA & $b / a_{\text {OXA-23 }}$ & $\mathrm{R}$ & $\mathrm{R}$ & $\mathrm{R}$ & $\mathrm{R}$ & $\mathrm{R}$ & $\mathrm{R}$ & $\mathrm{R}$ & $\mathrm{R}$ & $\mathrm{R}$ & $\mathrm{R}$ & $S$ & S \\
\hline N751 & 1 & NA & $b / a_{\mathrm{OXA}-23}$ & $\mathrm{R}$ & $\mathrm{R}$ & $\mathrm{R}$ & $\mathrm{R}$ & $\mathrm{R}$ & $\mathrm{R}$ & $\mathrm{R}$ & $\mathrm{R}$ & $\mathrm{R}$ & $\mathrm{R}$ & $S$ & S \\
\hline N752 & 1 & NA & $b / a_{\mathrm{OXA}-23}$ & $\mathrm{R}$ & $\mathrm{R}$ & $\mathrm{R}$ & R & R & $\mathrm{R}$ & $\mathrm{R}$ & $\mathrm{R}$ & $\mathrm{R}$ & $\mathrm{R}$ & 1 & S \\
\hline N756 & 1 & NA & $b / a_{\mathrm{OXA}-23}$ & $\mathrm{R}$ & $\mathrm{R}$ & $\mathrm{R}$ & 1 & $\mathrm{R}$ & $\mathrm{R}$ & $\mathrm{R}$ & $\mathrm{R}$ & $\mathrm{R}$ & $S$ & $S$ & S \\
\hline N762 & 1 & NA & $b / a_{\mathrm{OXA}-23}$ & $\mathrm{R}$ & $\mathrm{R}$ & $\mathrm{R}$ & $\mathrm{R}$ & $\mathrm{R}$ & $\mathrm{R}$ & $\mathrm{R}$ & $\mathrm{R}$ & R & $\mathrm{R}$ & 1 & S \\
\hline N764 & 1 & NA & $b l a_{\text {OXA-23 }}$ & $\mathrm{R}$ & $\mathrm{R}$ & $\mathrm{R}$ & $\mathrm{R}$ & $\mathrm{R}$ & $\mathrm{R}$ & $\mathrm{R}$ & $\mathrm{R}$ & $\mathrm{R}$ & $\mathrm{R}$ & I & S \\
\hline N1304 & 1 & NA & bla $\mathrm{OXA-72}$ & $\mathrm{R}$ & $\mathrm{R}$ & $\mathrm{R}$ & S & $\mathrm{R}$ & $\mathrm{R}$ & $\mathrm{R}$ & 1 & R & I & S & S \\
\hline N1307 & 1 & 2 & $b / a_{\text {OXA-72 }}$ & $\mathrm{R}$ & $\mathrm{R}$ & $\mathrm{R}$ & 1 & $\mathrm{R}$ & $\mathrm{R}$ & $\mathrm{R}$ & $\mathrm{R}$ & $\mathrm{R}$ & I & $\mathrm{S}$ & S \\
\hline N1309 & 1 & NA & bla ${ }_{\mathrm{OXA}-72}$ & $\mathrm{R}$ & $\mathrm{R}$ & $\mathrm{R}$ & $\mathrm{R}$ & $\mathrm{R}$ & $\mathrm{R}$ & $\mathrm{R}$ & I & R & $\mathrm{R}$ & S & S \\
\hline N1311 & 1 & NA & $b l a_{\text {OXA-72 }}$ & $\mathrm{R}$ & $\mathrm{R}$ & $\mathrm{R}$ & I & $\mathrm{R}$ & $\mathrm{R}$ & $\mathrm{R}$ & 1 & $\mathrm{R}$ & I & S & S \\
\hline N1315 & 1 & NA & $b / a_{\mathrm{OXA}-72}$ & $\mathrm{R}$ & $\mathrm{R}$ & $\mathrm{R}$ & $\mathrm{R}$ & $\mathrm{R}$ & $\mathrm{R}$ & $\mathrm{R}$ & $\mathrm{R}$ & $\mathrm{R}$ & $\mathrm{R}$ & $\mathrm{S}$ & S \\
\hline N743 & 1 & NA & $b l a_{\mathrm{OXA}-72}$ & $\mathrm{R}$ & $\mathrm{R}$ & $\mathrm{R}$ & $\mathrm{R}$ & $\mathrm{R}$ & $\mathrm{R}$ & $\mathrm{R}$ & $\mathrm{R}$ & $\mathrm{R}$ & $\mathrm{R}$ & $\mathrm{R}$ & S \\
\hline N745 & 1 & NA & $b / a_{\mathrm{OXA}-72}$ & $\mathrm{R}$ & $\mathrm{R}$ & $\mathrm{R}$ & I & $\mathrm{R}$ & $\mathrm{R}$ & $\mathrm{R}$ & $\mathrm{R}$ & $\mathrm{R}$ & I & I & S \\
\hline N750 & 1 & NA & $b^{b} a_{\mathrm{OXA}-72}$ & $\mathrm{R}$ & $\mathrm{R}$ & $\mathrm{R}$ & 1 & R & $\mathrm{R}$ & $\mathrm{R}$ & $\mathrm{R}$ & R & $\mathrm{R}$ & 1 & S \\
\hline N754 & 1 & NA & bla ${ }_{\mathrm{OXA}-72}$ & $\mathrm{R}$ & $\mathrm{R}$ & $\mathrm{R}$ & 1 & $\mathrm{R}$ & $\mathrm{R}$ & $\mathrm{R}$ & $\mathrm{R}$ & $\mathrm{R}$ & I & $S$ & S \\
\hline N1412 & 2 & NA & $b l a_{\mathrm{OXA}-23}$ & 1 & $\mathrm{R}$ & $\mathrm{R}$ & $\mathrm{R}$ & $\mathrm{R}$ & $\mathrm{R}$ & $\mathrm{R}$ & $\mathrm{R}$ & $\mathrm{R}$ & $\mathrm{R}$ & S & S \\
\hline N1419 & 2 & NA & $b l a_{\mathrm{OXA}-23}$ & I & $\mathrm{R}$ & $\mathrm{R}$ & $\mathrm{R}$ & $\mathrm{R}$ & $\mathrm{R}$ & $\mathrm{R}$ & $\mathrm{R}$ & $\mathrm{R}$ & $\mathrm{R}$ & $S$ & S \\
\hline N1420 & 2 & NA & $b / a_{\mathrm{OXA}-23}$ & $\mathrm{R}$ & $\mathrm{R}$ & $\mathrm{R}$ & $\mathrm{R}$ & $\mathrm{R}$ & $\mathrm{R}$ & $\mathrm{R}$ & $\mathrm{R}$ & $\mathrm{R}$ & $\mathrm{S}$ & $S$ & S \\
\hline N1424 & 2 & NA & $b / a_{\mathrm{OXA}-23}$ & 1 & $\mathrm{R}$ & $\mathrm{R}$ & $\mathrm{R}$ & $\mathrm{R}$ & $\mathrm{R}$ & $\mathrm{R}$ & $\mathrm{R}$ & $\mathrm{R}$ & $\mathrm{R}$ & $\mathrm{S}$ & S \\
\hline N1428 & 2 & NA & $b / a_{\mathrm{OXA}-23}$ & $\mathrm{R}$ & R & $\mathrm{R}$ & $\mathrm{R}$ & R & $\mathrm{R}$ & $\mathrm{R}$ & $\mathrm{R}$ & $\mathrm{R}$ & 1 & S & S \\
\hline N1447 & 2 & NA & $b / a_{\mathrm{OXA}-23}$ & $\mathrm{R}$ & $\mathrm{R}$ & $\mathrm{R}$ & R & $\mathrm{R}$ & $\mathrm{R}$ & $\mathrm{R}$ & 1 & $\mathrm{R}$ & $\mathrm{R}$ & $\mathrm{S}$ & S \\
\hline N1449 & 2 & NA & bla OXA-23 & $\mathrm{R}$ & $\mathrm{R}$ & $\mathrm{R}$ & $\mathrm{R}$ & $\mathrm{R}$ & $\mathrm{R}$ & $\mathrm{R}$ & I & $\mathrm{R}$ & $\mathrm{R}$ & $S$ & S \\
\hline
\end{tabular}


Table 4 The Rep-PCR type, MLST type, presence of carbapenemase genes and antimicrobial resistance profile of 101 Acinetobacter baumannii isolates (Continued)

\begin{tabular}{|c|c|c|c|c|c|c|c|c|c|c|c|c|c|c|c|}
\hline \multirow{2}{*}{$\begin{array}{l}\text { Isolate } \\
\text { ID }\end{array}$} & \multirow{2}{*}{$\begin{array}{l}\text { Rep-PCR } \\
\text { type }\end{array}$} & \multirow{2}{*}{$\begin{array}{l}\text { MLST } \\
\text { type }\end{array}$} & \multirow{2}{*}{$\begin{array}{l}\text { Carbapenemase } \\
\text { genes }\end{array}$} & \multicolumn{12}{|c|}{ Antimicrobial resistance profile } \\
\hline & & & & $\overline{I M P}$ & MEM & CAZ & FEP & AMK & GEN & CIP & LVX & TZP & SAM & $\mathrm{MH}$ & $\mathrm{COL}$ \\
\hline N1372 & 2 & NA & $b / a_{\text {OXA }-72}$ & $\mathrm{R}$ & $\mathrm{R}$ & $\mathrm{R}$ & 1 & $\mathrm{R}$ & $\mathrm{R}$ & $\mathrm{R}$ & $\mathrm{R}$ & $\mathrm{R}$ & $\mathrm{R}$ & I & S \\
\hline N1411 & 2 & NA & $b / a_{\mathrm{OXA}-72}$ & $\mathrm{R}$ & $\mathrm{R}$ & $\mathrm{R}$ & । & $\mathrm{R}$ & $\mathrm{R}$ & $\mathrm{R}$ & $\mathrm{R}$ & $\mathrm{R}$ & $\mathrm{R}$ & $\mathrm{S}$ & S \\
\hline N1425 & 2 & 2 & $b / a_{\mathrm{OXA}-72}$ & $\mathrm{R}$ & $\mathrm{R}$ & $\mathrm{R}$ & S & $\mathrm{R}$ & $\mathrm{R}$ & $\mathrm{R}$ & $\mathrm{R}$ & $\mathrm{R}$ & । & $\mathrm{S}$ & S \\
\hline N1446 & 2 & NA & bla OXA-72 & $\mathrm{R}$ & $\mathrm{R}$ & $\mathrm{R}$ & । & $\mathrm{R}$ & $\mathrm{R}$ & $\mathrm{R}$ & $\mathrm{R}$ & $\mathrm{R}$ & $\mathrm{R}$ & $S$ & S \\
\hline N1451 & 2 & NA & $b / a_{\text {OXA-72 }}$ & $\mathrm{R}$ & $\mathrm{R}$ & $\mathrm{R}$ & $\mathrm{R}$ & $\mathrm{R}$ & $\mathrm{R}$ & $\mathrm{R}$ & $\mathrm{R}$ & $\mathrm{R}$ & $\mathrm{R}$ & I & S \\
\hline N1348 & 3 & NA & $b / a_{\mathrm{OXA}-72}$ & $\mathrm{R}$ & $\mathrm{R}$ & $\mathrm{R}$ & 1 & $\mathrm{R}$ & $\mathrm{R}$ & $\mathrm{R}$ & $\mathrm{R}$ & R & $\mathrm{R}$ & $S$ & S \\
\hline N1349 & 3 & NA & $b / a_{\text {OXA-72 }}$ & $\mathrm{R}$ & $\mathrm{R}$ & $\mathrm{R}$ & I & $\mathrm{R}$ & $\mathrm{R}$ & $\mathrm{R}$ & $\mathrm{R}$ & $\mathrm{R}$ & $\mathrm{R}$ & $S$ & S \\
\hline N1368 & 3 & NA & $b / a_{\mathrm{OXA}-72}$ & $\mathrm{R}$ & $\mathrm{R}$ & $\mathrm{R}$ & 1 & $\mathrm{R}$ & $\mathrm{R}$ & $\mathrm{R}$ & $\mathrm{R}$ & $\mathrm{R}$ & $\mathrm{R}$ & $S$ & S \\
\hline N1376 & 3 & NA & bla OXA-72 & $\mathrm{R}$ & $\mathrm{R}$ & $\mathrm{R}$ & $S$ & $\mathrm{R}$ & $\mathrm{R}$ & $\mathrm{R}$ & $\mathrm{R}$ & $\mathrm{R}$ & $\mathrm{R}$ & $S$ & S \\
\hline N1378 & 3 & NA & $b / a_{\text {OXA-72 }}$ & $\mathrm{R}$ & $\mathrm{R}$ & $\mathrm{R}$ & I & $\mathrm{R}$ & $\mathrm{R}$ & $\mathrm{R}$ & $\mathrm{R}$ & $\mathrm{R}$ & $\mathrm{R}$ & $S$ & S \\
\hline N1421 & 3 & 2 & $b / a_{\mathrm{OXA}-72}$ & $\mathrm{R}$ & $\mathrm{R}$ & $\mathrm{R}$ & I & $\mathrm{R}$ & $\mathrm{R}$ & $\mathrm{R}$ & $\mathrm{R}$ & $\mathrm{R}$ & I & S & S \\
\hline N1427 & 3 & NA & $b / a_{\mathrm{OXA}-72}$ & $\mathrm{R}$ & $\mathrm{R}$ & $\mathrm{R}$ & I & $\mathrm{R}$ & $\mathrm{R}$ & $\mathrm{R}$ & $\mathrm{R}$ & $\mathrm{R}$ & $\mathrm{R}$ & $S$ & S \\
\hline N1429 & 3 & NA & $b l a_{\text {OXA-72 }}$ & $\mathrm{R}$ & $\mathrm{R}$ & $\mathrm{R}$ & I & $S$ & $S$ & $\mathrm{R}$ & 1 & R & $\mathrm{R}$ & $S$ & S \\
\hline N1432 & 3 & NA & $b l a_{\mathrm{OXA}-72}$ & $\mathrm{R}$ & $\mathrm{R}$ & $\mathrm{R}$ & I & $\mathrm{R}$ & $\mathrm{R}$ & $\mathrm{R}$ & $\mathrm{R}$ & $\mathrm{R}$ & $S$ & S & S \\
\hline N1436 & 3 & NA & $b / a_{\text {OXA-72 }}$ & $\mathrm{R}$ & $\mathrm{R}$ & $\mathrm{R}$ & $\mathrm{R}$ & $\mathrm{R}$ & $\mathrm{R}$ & $\mathrm{R}$ & $\mathrm{R}$ & $R$ & $\mathrm{R}$ & $S$ & S \\
\hline N1437 & 3 & NA & $b / a_{\mathrm{OXA}-72}$ & $\mathrm{R}$ & $\mathrm{R}$ & $\mathrm{R}$ & 1 & $\mathrm{R}$ & $\mathrm{R}$ & $\mathrm{R}$ & $\mathrm{R}$ & $\mathrm{R}$ & $\mathrm{R}$ & S & S \\
\hline N1365 & 4 & NA & bla OXA-72 & $\mathrm{R}$ & $\mathrm{R}$ & $\mathrm{R}$ & । & $\mathrm{R}$ & $\mathrm{R}$ & $\mathrm{R}$ & $\mathrm{R}$ & $\mathrm{R}$ & $\mathrm{R}$ & $S$ & S \\
\hline N1366 & 4 & NA & $b l a_{\mathrm{OXA}-72}$ & $\mathrm{R}$ & $\mathrm{R}$ & $\mathrm{R}$ & $\mathrm{R}$ & $\mathrm{R}$ & $\mathrm{R}$ & $\mathrm{R}$ & $\mathrm{R}$ & $\mathrm{R}$ & $\mathrm{R}$ & S & S \\
\hline N1422 & 4 & 2 & $b / a_{\mathrm{OXA}-72}$ & $\mathrm{R}$ & $\mathrm{R}$ & $\mathrm{R}$ & $\mathrm{S}$ & $\mathrm{R}$ & $\mathrm{R}$ & $\mathrm{R}$ & I & $\mathrm{R}$ & I & $\mathrm{S}$ & S \\
\hline N1434 & 4 & NA & $b / a_{\mathrm{OXA}-72}$ & $\mathrm{R}$ & $\mathrm{R}$ & $\mathrm{R}$ & I & $\mathrm{R}$ & $\mathrm{R}$ & $\mathrm{R}$ & $\mathrm{R}$ & $\mathrm{R}$ & $\mathrm{R}$ & $\mathrm{S}$ & S \\
\hline N1361 & 5 & NA & $b / a_{\text {OXA-72 }}$ & $\mathrm{R}$ & $\mathrm{R}$ & $\mathrm{R}$ & I & $\mathrm{R}$ & $\mathrm{R}$ & $\mathrm{R}$ & $\mathrm{R}$ & $\mathrm{R}$ & $\mathrm{R}$ & $S$ & S \\
\hline N1375 & 5 & NA & $b / a_{\mathrm{OXA}-72}$ & $\mathrm{R}$ & $\mathrm{R}$ & $\mathrm{R}$ & $\mathrm{R}$ & $\mathrm{R}$ & 1 & $\mathrm{R}$ & $\mathrm{R}$ & $\mathrm{R}$ & $\mathrm{R}$ & $S$ & S \\
\hline N1380 & 5 & NA & $b l a_{\mathrm{OXA}-72}$ & $\mathrm{R}$ & $\mathrm{R}$ & $\mathrm{R}$ & $S$ & $\mathrm{R}$ & $\mathrm{R}$ & $\mathrm{R}$ & $\mathrm{R}$ & $\mathrm{R}$ & $\mathrm{R}$ & S & S \\
\hline N1381 & 5 & NA & bla oxA-72 & $\mathrm{R}$ & $\mathrm{R}$ & $\mathrm{R}$ & $S$ & $\mathrm{R}$ & $\mathrm{R}$ & $\mathrm{R}$ & $\mathrm{R}$ & $\mathrm{R}$ & $\mathrm{R}$ & $S$ & S \\
\hline N1397 & 5 & 2 & $b / a_{\mathrm{OXA}-72}$ & $\mathrm{R}$ & $\mathrm{R}$ & $\mathrm{R}$ & I & $\mathrm{R}$ & $\mathrm{R}$ & $\mathrm{R}$ & $\mathrm{R}$ & R & $\mathrm{R}$ & $\mathrm{S}$ & S \\
\hline N1400 & 5 & NA & $b l a_{\text {OXA-72 }}$ & $\mathrm{R}$ & $\mathrm{R}$ & $\mathrm{R}$ & I & $\mathrm{R}$ & $\mathrm{R}$ & $\mathrm{R}$ & $\mathrm{R}$ & $\mathrm{R}$ & $\mathrm{R}$ & $S$ & S \\
\hline N1350 & 6 & NA & $b l a_{\mathrm{OXA}-72}$ & $\mathrm{R}$ & $\mathrm{R}$ & $\mathrm{R}$ & I & $\mathrm{R}$ & $\mathrm{R}$ & $\mathrm{R}$ & $\mathrm{R}$ & $\mathrm{R}$ & $\mathrm{R}$ & S & S \\
\hline N1353 & 6 & 2 & $b / a_{\text {OXA-72 }}$ & $\mathrm{R}$ & $\mathrm{R}$ & $\mathrm{R}$ & $\mathrm{R}$ & $\mathrm{R}$ & $\mathrm{R}$ & $\mathrm{R}$ & $\mathrm{R}$ & $\mathrm{R}$ & $\mathrm{R}$ & $\mathrm{S}$ & S \\
\hline N1370 & 6 & NA & bla ${ }_{\mathrm{OXA}-72}$ & $\mathrm{R}$ & $\mathrm{R}$ & $\mathrm{R}$ & I & $\mathrm{R}$ & $\mathrm{R}$ & $\mathrm{R}$ & $\mathrm{R}$ & R & $\mathrm{R}$ & I & S \\
\hline N1391 & 7 & 2 & $b l a_{\text {OXA-72 }}$ & $\mathrm{R}$ & $\mathrm{R}$ & $\mathrm{R}$ & $\mathrm{R}$ & $\mathrm{R}$ & $\mathrm{R}$ & $\mathrm{R}$ & $\mathrm{R}$ & R & $\mathrm{R}$ & S & S \\
\hline N1357 & 8 & 2 & $b / a_{\mathrm{OXA}-72}$ & $\mathrm{R}$ & $\mathrm{R}$ & $\mathrm{S}$ & $\mathrm{S}$ & $S$ & $\mathrm{R}$ & $\mathrm{R}$ & $\mathrm{R}$ & 1 & $\mathrm{~S}$ & $\mathrm{~S}$ & S \\
\hline N1374 & 8 & NA & $b l a_{\mathrm{OXA}-72}$ & $\mathrm{R}$ & $\mathrm{R}$ & $\mathrm{R}$ & 1 & $\mathrm{R}$ & $\mathrm{R}$ & $\mathrm{R}$ & $\mathrm{R}$ & $\mathrm{R}$ & $\mathrm{R}$ & $\mathrm{S}$ & S \\
\hline N1379 & 8 & NA & $b / a_{\mathrm{OXA}-72}$ & $\mathrm{R}$ & $\mathrm{R}$ & $\mathrm{R}$ & I & $\mathrm{R}$ & $\mathrm{R}$ & $\mathrm{R}$ & $\mathrm{R}$ & $\mathrm{R}$ & $\mathrm{R}$ & $S$ & S \\
\hline N1392 & 8 & NA & $b^{b} a_{\mathrm{OXA}-72}$ & $\mathrm{R}$ & R & $\mathrm{R}$ & $\mathrm{S}$ & R & $\mathrm{R}$ & $\mathrm{R}$ & $\mathrm{R}$ & $\mathrm{R}$ & $\mathrm{R}$ & S & S \\
\hline N1409 & 8 & NA & bla ${ }_{\mathrm{OXA}-72}$ & $\mathrm{R}$ & $\mathrm{R}$ & $\mathrm{R}$ & $S$ & $\mathrm{R}$ & $\mathrm{R}$ & $\mathrm{R}$ & I & $\mathrm{R}$ & I & $S$ & S \\
\hline N1382 & 9 & 2 & $b l a_{\mathrm{OXA}-72}$ & $\mathrm{R}$ & R & $\mathrm{R}$ & $\mathrm{S}$ & R & R & $\mathrm{R}$ & $\mathrm{R}$ & $\mathrm{R}$ & $\mathrm{R}$ & S & S \\
\hline N1415 & 10 & NA & None & $S$ & $S$ & $S$ & $S$ & $S$ & $S$ & $S$ & S & S & $S$ & S & S \\
\hline N1441 & 11 & NA & None & $S$ & $S$ & $S$ & $S$ & $S$ & $\mathrm{R}$ & $S$ & S & S & $\mathrm{R}$ & $S$ & S \\
\hline N1443 & 12 & NA & None & $S$ & $\mathrm{~S}$ & $\mathrm{~S}$ & $\mathrm{~S}$ & $\mathrm{~S}$ & $\mathrm{~S}$ & $\mathrm{~S}$ & S & S & $\mathrm{S}$ & $S$ & S \\
\hline N1408 & 13 & NA & None & S & $S$ & $\mathrm{~S}$ & $S$ & $S$ & $S$ & $S$ & $S$ & S & $S$ & $\mathrm{~S}$ & S \\
\hline N1306 & 14 & NA & None & $S$ & $\mathrm{~S}$ & $\mathrm{~S}$ & $\mathrm{~S}$ & $\mathrm{~S}$ & $\mathrm{~S}$ & $\mathrm{~S}$ & S & S & $\mathrm{S}$ & $\mathrm{S}$ & S \\
\hline N1414 & 15 & NA & None & $S$ & $S$ & $S$ & $S$ & $S$ & $S$ & $S$ & $\mathrm{~S}$ & S & $S$ & $S$ & S \\
\hline
\end{tabular}


Table 4 The Rep-PCR type, MLST type, presence of carbapenemase genes and antimicrobial resistance profile of 101 Acinetobacter baumannii isolates (Continued)

\begin{tabular}{|c|c|c|c|c|c|c|c|c|c|c|c|c|c|c|c|}
\hline \multirow{2}{*}{$\begin{array}{l}\text { Isolate } \\
\text { ID }\end{array}$} & \multirow{2}{*}{$\begin{array}{l}\text { Rep-PCR } \\
\text { type }\end{array}$} & \multirow{2}{*}{$\begin{array}{l}\text { MLST } \\
\text { type }\end{array}$} & \multirow{2}{*}{$\begin{array}{l}\text { Carbapenemase } \\
\text { genes }\end{array}$} & \multicolumn{12}{|c|}{ Antimicrobial resistance profile } \\
\hline & & & & $\mathrm{IMP}$ & MEM & CAZ & FEP & AMK & GEN & CIP & LVX & TZP & SAM & $\mathrm{MH}$ & $\mathrm{COL}$ \\
\hline N1363 & 16 & NA & None & $S$ & $S$ & $S$ & $S$ & $S$ & $S$ & $S$ & $\mathrm{~S}$ & $S$ & $S$ & $S$ & $S$ \\
\hline N1389 & 17 & NA & None & $S$ & $S$ & $S$ & $S$ & S & S & $S$ & $S$ & $S$ & $S$ & $S$ & $S$ \\
\hline N1405 & 18 & NA & None & $S$ & $S$ & $S$ & $S$ & S & S & $S$ & $\mathrm{~S}$ & $S$ & $S$ & $S$ & $S$ \\
\hline N1406 & 18 & NA & None & $S$ & $\mathrm{~S}$ & S & $\mathrm{S}$ & S & S & $S$ & $S$ & $S$ & $S$ & $\mathrm{~S}$ & $\mathrm{R}$ \\
\hline N1377 & 19 & NA & None & $S$ & $S$ & $\mathrm{R}$ & $\mathrm{R}$ & $\mathrm{R}$ & $\mathrm{R}$ & $\mathrm{R}$ & $\mathrm{R}$ & $\mathrm{R}$ & $\mathrm{R}$ & $S$ & $S$ \\
\hline N1390 & 20 & NA & $b / a_{\mathrm{OXA}-58}$ & $\mathrm{~S}$ & $S$ & $S$ & $S$ & S & $\mathrm{R}$ & $S$ & $\mathrm{~S}$ & $S$ & $S$ & $S$ & $S$ \\
\hline N1448 & 21 & NA & None & S & $S$ & $S$ & $S$ & S & $\mathrm{R}$ & $\mathrm{R}$ & $\mathrm{R}$ & $S$ & $S$ & $S$ & $\mathrm{R}$ \\
\hline N1354 & 22 & NA & None & S & $S$ & $S$ & $S$ & S & S & $S$ & $S$ & $S$ & $\mathrm{~S}$ & $\mathrm{~S}$ & $\mathrm{~S}$ \\
\hline N746 & 23 & NA & None & $S$ & $S$ & $S$ & $S$ & S & S & $S$ & $S$ & $S$ & I & $S$ & $S$ \\
\hline N1364 & 24 & NA & None & S & $S$ & $S$ & $S$ & S & S & $S$ & $S$ & $S$ & $S$ & $\mathrm{~S}$ & $\mathrm{~S}$ \\
\hline N1393 & 25 & NA & None & $S$ & $S$ & $S$ & $S$ & S & $S$ & $S$ & $S$ & $S$ & $S$ & $S$ & $S$ \\
\hline N1351 & 26 & NA & None & $S$ & $S$ & $S$ & $S$ & S & S & $S$ & $S$ & $S$ & $S$ & $S$ & $S$ \\
\hline N1399 & 27 & NA & None & $S$ & $S$ & $S$ & $S$ & S & $S$ & $S$ & $S$ & $S$ & $S$ & $S$ & $S$ \\
\hline N1305 & 28 & NA & None & S & $S$ & $S$ & $S$ & $S$ & S & $S$ & $S$ & $S$ & $S$ & $S$ & $S$ \\
\hline N1387 & 29 & NA & None & $S$ & $S$ & $S$ & $S$ & $S$ & $S$ & $S$ & $S$ & $S$ & $S$ & $S$ & $S$ \\
\hline N744 & 30 & NA & None & $S$ & $S$ & $S$ & $S$ & $S$ & $S$ & $S$ & $S$ & $S$ & $S$ & $S$ & $\mathrm{R}$ \\
\hline N1423 & 31 & NA & None & $S$ & $S$ & $S$ & $S$ & S & S & $S$ & $S$ & $S$ & $S$ & $S$ & $S$ \\
\hline
\end{tabular}

NA not available, IMP imipenem, MEM meropenem, CAZ ceftazidime, FEP cefepime, AMK amikacin, GEN gentamicin, CIP ciprofloxacin, LVX levofloxacin, TZP piperacillin-tazobactam, $S A M$ ampicillin-sulbactam, $M H$ minocyline, $C O L$ colistin, $R$ resistant, $I$ intermediate, $S$ susceptible

were distributed in the 9 Rep-PCR types. Minimum spanning tree analysis of Rep-PCR patterns showed that all the 78 CRAB isolates were clustered into one clone complex, while most of the CSAB isolates were not connected to each other (Fig. 1). MLST analysis showed that all the 9 representative OXA-72 isolates in this study belong to ST2 (Table 4).

A literature review of previous published MLST data showed that there were at least 19 ST types (Bartual scheme) for 29 OXA-24/40-like producing Acinetobacter spp. isolates from 11 countries and 21 ST types (Pasteur scheme) for 52 OXA-24/40-like producing Acinetobacter spp. isolates from 15 countries. Minimum spanning tree analysis of these isolates based on two different MLST schemes was shown in Fig. 2, which suggested that $\mathrm{CC} 92^{\mathrm{B}} / \mathrm{CC} 2^{\mathrm{P}}$ represented the predominant clone for the OXA-24/40-like producing Acinetobacter spp. isolates from around the world.

\section{Discussions}

Carbapenem-resistant Acinetobacter spp. (mainly CRAB) are increasingly recognized as major nosocomial pathogens and considered to be serious threat for human health by US Centers for Disease Control and Prevention and World Health Organization [24, 25]. OXA-23-like carbapenemases were the main reason for the high prevalence and wide dissemination of CRAB from many parts of the world, including China [14, 26, 27]. OXA-24/40-like carbapenemases, which have been reported to be associated with outbreak of nosocomial infection in United States, Spain, Turkey and Ecuador [28-31], accounted for only a small part of CRAB in China [14, 32]. OXA-72, which was first identified in 2004 in an A. baumannii isolate from Thailand, belonged to one of the most important variant of OXA-24/40-like carbapenemases [29]. This study reported firstly a high prevalence and clonal dissemination of OXA-72-producing A. baumannii in a hospital from northeastern China.

Carbapenem resistance in A. baumannii was not significantly associated with 14-day mortality in this study (Table 1), which is in accordance with previous studies $[33,34]$. However, carbapenem resistance limits the available therapeutic agents, makes the infection difficult to treat, and might be associated with an additional cost of hospitalization [34]. In this study, there was no significant difference over length of hospital stay for carbapenem resistance and susceptible $A$. baumannii, which might be related with the limited sample size.

In vitro antimicrobial susceptibility testing showed that OXA-72-producing A. baumannii and OXA-23-producing A. baumannii exhibited similar multidrug resistance profile, suggesting that they could not be differentiated through 


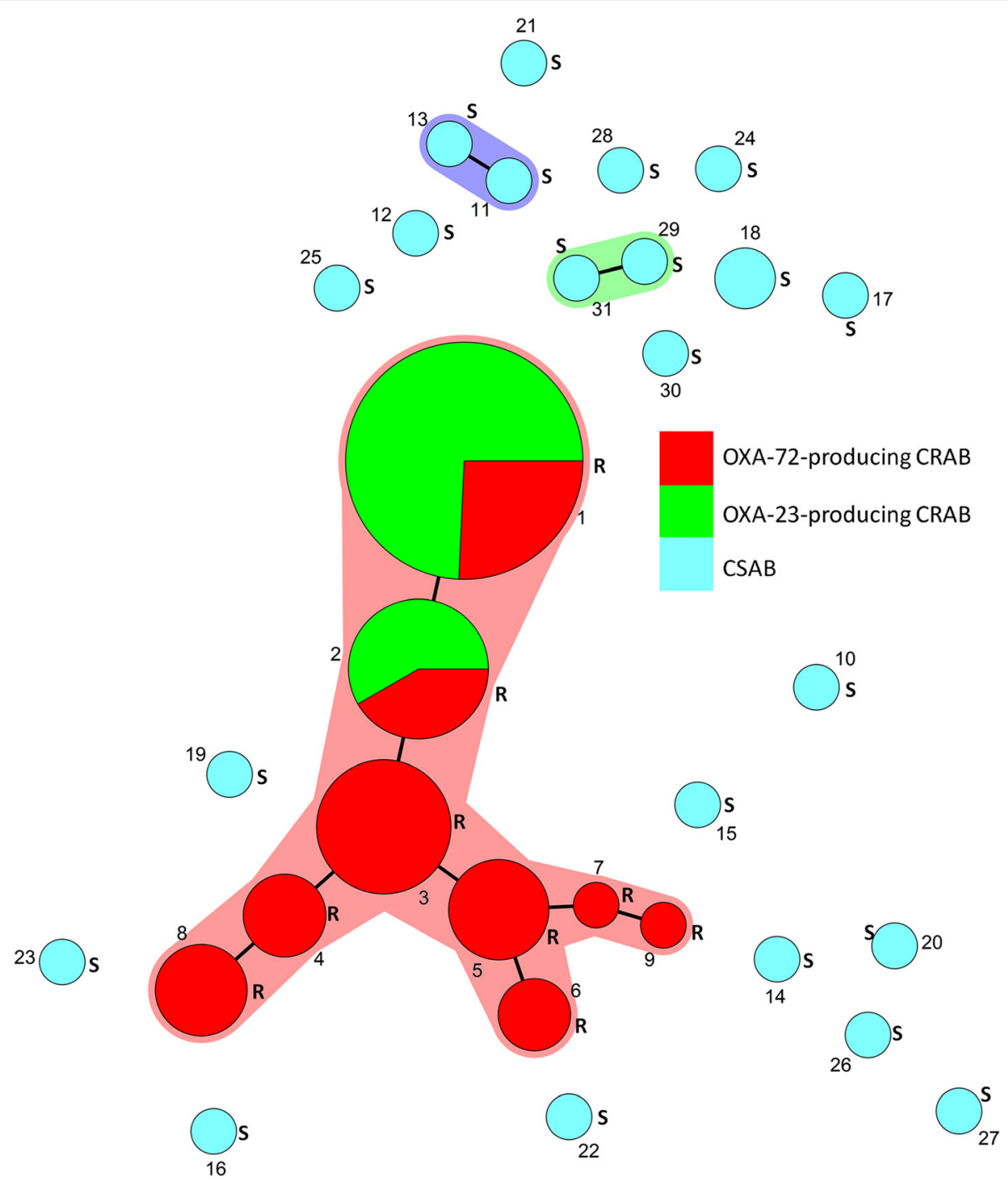

Fig. 1 Minimum spanning tree of 101 Acinetobacter baumannii isolates based on Rep-PCR patterns. Each circle represents one unique genotype. OXA-72-producing carbapenem-resistant A. baumannii (CRAB), OXA-23-producing CRAB and carbapenem-susceptible A. baumannii (CSAB) isolates are indicated with red, green, and blue colors, respectively. The size of each circle corresponds to the number of isolates. The lines connecting the circles indicate those patterns that differ by a single band. R: carbapenem-resistant strains. S: carbapenem-susceptible strains

detection of antimicrobial phenotype. The risk factor analyses implicated that admitted into ICU and length of ICU stay were the most important risk factors for carriage of OXA-72-producing A. baumannii and OXA-23-producing A. baumannii, as ICU patients are always critical ill and subjected to a lot of risk factors for the acquisition of multidrug resistance organisms (MDROs) [35]. When ICU stay was removed for multivariable analyses, nasogastric intubation and carbapenem use were significantly associated with acquisition of both classes of CRAB, which is in accordance with previous studies [36-38]. The reason for why urinary catheter was significantly associated with carriage of OXA-23-producing A. baumannii, but not OXA-72-producing A. baumannii deserves further investigation. One possible explanation was that the complex conditions and combined therapy of ICU patients compromised the accuracy of multiple logistics analysis, urinary catheter might be just an indicator for critical ill patients who have a high probability of acquiring certain MDROs through contaminated environment or nursing behavior.

$\mathrm{CC} 92^{\mathrm{B}} / \mathrm{CC} 2^{\mathrm{P}}$ was by far the largest and most widely distributed $A$. baumannii clone in the world, especially among OXA-23-producing A. baumannii [27, 39, 40]. Although not so widely disseminated, $\mathrm{CC} 92^{\mathrm{B}} / \mathrm{CC} 2^{\mathrm{P}}$ was still the most important clone in OXA-24/40-producing A. baumannii 


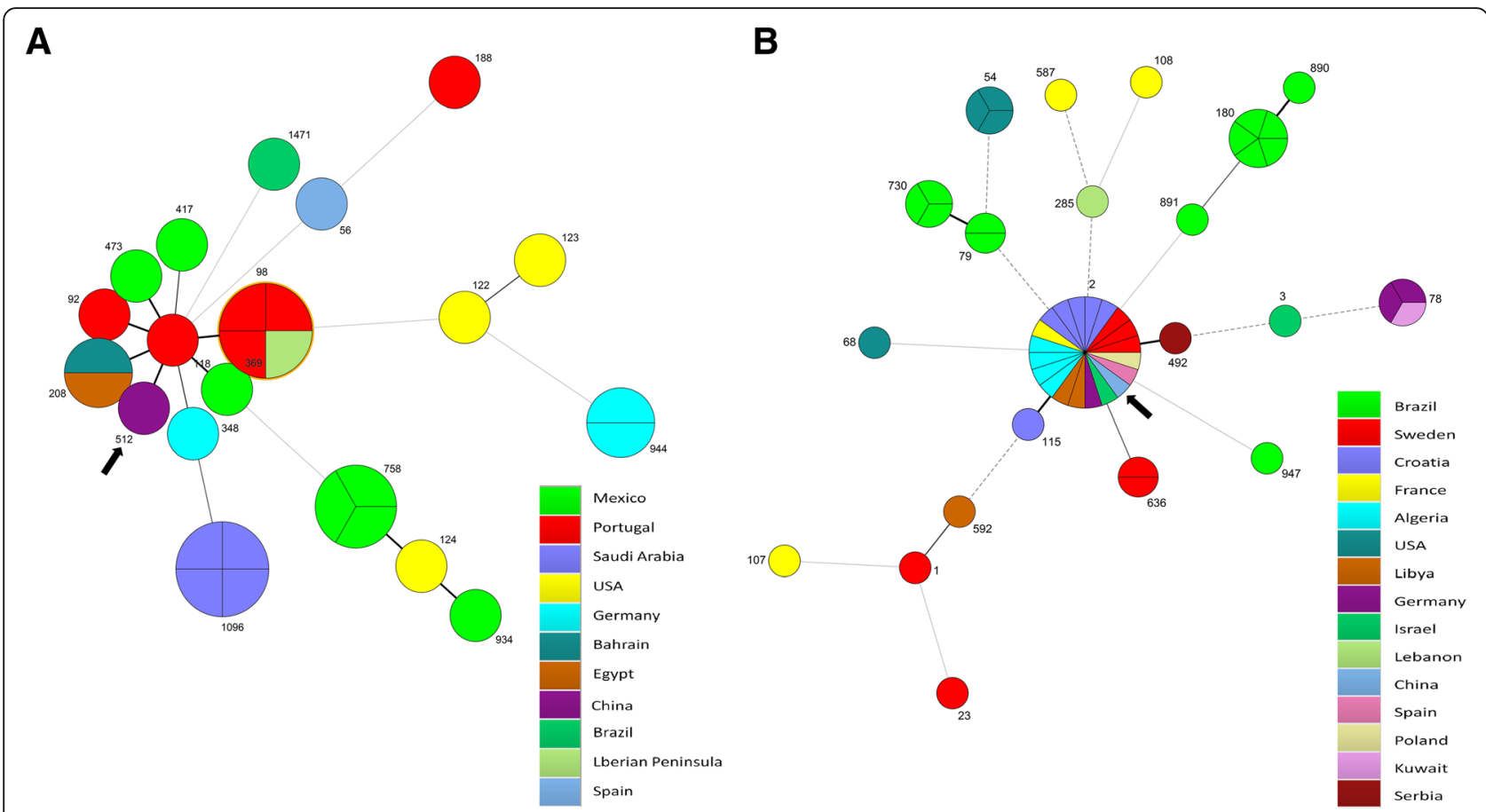

Fig. 2 Minimum spanning tree analysis of bla OXA-72-positive Acinetobacter baumannii isolates based on multilocus sequence typing (MLST) data from published literatures. The left panel (a) showed the results from 'Bartual' scheme, the right panel (b) showed the results from 'Pasteur' scheme. Each circle represents an independent sequence type (ST). The size of each circle corresponds to the number of isolates. The lines connecting the circles indicate the relationship between different STs. Black arrows are used to indicate the strains originated from China

(Fig. 2). The Rep-PCR and MLST analysis of A. baumannii in this study suggested that OXA-72-producing and OXA-23-producing A. baumannii isolates were genetically related and belonged to the same clone, $\mathrm{CC} 92^{\mathrm{B}} / \mathrm{CC} 2^{\mathrm{P}}$. It seems that OXA-72-producing A. baumannii has already become endemic in the ICU since 2014, as most of these isolates were continuously cultured without obvious clustering of isolation time. Enhanced infection control measures, such as hand hygiene education programs, environmental cleaning, antimicrobial stewardship, contact precautions [41], have to be implemented in ICU of this hospital in order to reduce the wide spread of high risk clone, $\mathrm{CC} 92^{\mathrm{B}} / \mathrm{CC} 2^{\mathrm{P}}$, which represents the most prevalent clone of $C R A B$ in Chinese hospitals.

There are some limitations for this study. The first is the inclusion criteria of Acb complex strains, it has been demonstrated that a single patient may have more than one genetic type of Acinetobacter [42, 43]. To avoid the problem of duplicate data, this study adopted a simple inclusion method of allowing only a single isolate per patient. it might limit the ability to monitor the dynamic changes and complex conditions in patients who may be at particular risk of acquiring antibiotic resistant strains through cross-infection or the development of resistance during antibiotic treatment [44]. Another limitation comes from the design of this study, as this is just an one-center study, the epidemiological characterizations of OXA-72 strains in this study might not be generalized to other healthcare settings in China.

\section{Conclusions}

This study described firstly a high prevalence of OXA-72-producing A. baumannii in ICU of a Chinese hospital, which have circulated in this ICU through clonal dissemination for at least two years. Strict infection control measures must be implemented to contain the ongoing dissemination of OXA carbapenemases-producing A. baumannii in Chinese ICUs.

\section{Abbreviations}

Acb: Acinetobacter calcoaceticus-A. baumannii; CC: Clone complex:

CRAB: Carbapenem-resistant A. baumannii; CSAB: Carbapenem-susceptible A. baumannii; DI: Index of diversity; ICU: Intensive care unit; MDROs: Multidrug resistance organisms; MLST: Multilocus sequence typing; ST: Sequence type

\section{Funding}

The study was supported by a grant from the National Key Program for Infectious Diseases of China (2018ZX10733402), Beijing Natural Science Foundation (7172157) and the Beijing Nova Program (Z181100006218107).

\section{Availability of data and materials}

The datasets used and/or analyzed during the current study available from the corresponding author on reasonable request.

\section{Authors' contributions}

$\mathrm{LH}, \mathrm{HL}$, JS and HG conceptualized and designed the study. YY, LL, GQ, XH, $\mathrm{ST}, \mathrm{JZ}, \mathrm{FC}$ were involved in the data collection, generation, and performed 
laboratory analysis. YC, YY and LL analyzed and interpreted the data, drafted the manuscript. LH and HG revised the manuscript critically for important intellectual content. All authors read and approved the final manuscript.

\section{Ethics approval and consent to participate}

The study was approved by the institutional ethics committees of the Academy of Military Medical Sciences of the Chinese People's Liberation Army, Beijing, China. Because the study was epidemiological without any interventions and all the data were collected and analyzed anonymously, the requirement for informed consent was waived.

\section{Consent for publication}

Not applicable.

\section{Competing interests}

The authors declare that they have no competing interests.

\section{Publisher's Note}

Springer Nature remains neutral with regard to jurisdictional claims in published maps and institutional affiliations.

\section{Author details}

'Department of Hospital Infection Control, Chinese PLA Institute for Disease Control and Prevention, 20\# Dongda Str, Beijing 100071, China. ${ }^{2}$ School of Public Health, Shenyang Medical College, Shenyang, China. ${ }^{3}$ The 202nd Hospital of PLA, Shenyang 110003, China. ${ }^{4}$ Laboratory of Tropical Biomedicine Technology, School of Tropical Medicine and Laboratory Medicine, Hainan Medical University, Haikou, China. ${ }^{5}$ Department of Clinical Microbiology, The 202nd Hospital of PLA, Shenyang, China. ${ }^{6}$ Department of Infection Prevention and Hospital Hygiene, Faculty of Medicine, University of Freiburg, Freiburg, Germany. ${ }^{7}$ Department of Medical Microbiology, University Medical Center Groningen, Rijksuniversteit Groningen, Groningen, The Netherlands. ${ }^{8}$ Department of Hospital Infection Control, The 202nd Hospital of PLA, Shenyang 110003, China. ${ }^{9}$ Chinese PLA 202 Hospital, Shenyang 110003, China.

\section{Received: 17 November 2017 Accepted: 23 August 2018} Published online: 29 September 2018

\section{References}

1. Lima AL, Oliveira PR, Paula AP. Acinetobacter infection. N Engl J Med. 2008; 358(12):1271-81.

2. Peleg AY, Seifert $H$, Paterson DL. Acinetobacter baumannii: emergence of a successful pathogen. Clin Microbiol Rev. 2008;21(3):538-82.

3. Nemec A, Krizova L, Maixnerova M, van der Reijden TJ, Deschaght P, Passet $\checkmark$, et al. Genotypic and phenotypic characterization of the Acinetobacter calcoaceticus-Acinetobacter baumannii complex with the proposal of Acinetobacter pittii sp. nov. (formerly Acinetobacter genomic species 3) and Acinetobacter nosocomialis sp. nov. (formerly Acinetobacter genomic species 13TU). Res Microbiol. 2011;162(4):393-404.

4. Antunes LC, Visca P, Towner KJ. Acinetobacter baumannii: evolution of a global pathogen. Pathog and Dis. 2014;71(3):292-301.

5. Doi Y, Murray GL, Peleg AY. Acinetobacter baumannii: evolution of antimicrobial resistance-treatment options. Semin Respir Rrit Care Med. 2015;36(1):85-98.

6. Mera RM, Miller LA, Amrine-Madsen H, Sahm DF. Acinetobacter baumannii 2002-2008: increase of carbapenem-associated multiclass resistance in the United States. Microb Drug Resist. 2010;16(3):209-15.

7. Xiao YH, Giske CG, Wei ZQ, Shen P, Heddini A, Li L. Epidemiology and characteristics of antimicrobial resistance in China. Drug Resist Updat. 2011;14(4-5):236-50.

8. Hu FP, Guo Y, Zhu DM, Wang F, Jiang XF, Xu YC, et al. Resistance trends among clinical isolates in China reported from CHINET surveillance of bacterial resistance, 2005-2014. Clin Microbiol Infect. 2016;22(Suppl 1):S9-14.

9. Docquier JD, Mangani S. Structure-function relationships of class D Carbapenemases. Curr Drug Targets. 2016;17(9):1061-71.

10. Antunes NT, Lamoureaux TL, Toth M, Stewart NK, Frase H, Vakulenko SB. Class D -lactamases: are they all Carbapenemases? Antimicrob Agents Chemother. 2014;58(4):2119-25.

11. Evans BA, Amyes SG. OXA beta-lactamases. Clin Microbiol Rev. 2014;27(2): 241-63.
12. Bou G, Oliver A, Martinez-Beltran J. OXA-24, a novel class D beta-lactamase with carbapenemase activity in an Acinetobacter baumannii clinical strain. Antimicrob Agents Chemother. 2000;44(6):1556-61.

13. Mendes RE, Bell JM, Turnidge JD, Castanheira M, Jones RN. Emergence and widespread dissemination of OXA-23, $-24 / 40$ and -58 carbapenemases among Acinetobacter spp. in Asia-Pacific nations: report from the SENTRY Surveillance Program. J Agents Chemother. 2009;63(1):55-9.

14. Ji S, Chen Y, Ruan Z, Fu Y, Ji J, Fu Y, et al. Prevalence of carbapenemhydrolyzing class $D$ beta-lactamase genes in Acinetobacter spp. isolates in China. Eur J Clin Microbiol Infect Dis. 2014;33(6):989-97.

15. Higgins $P G$, Lehmann $M$, Wisplinghoff $H$, Seifert $H$. gyrB multiplex PCR to differentiate between Acinetobacter calcoaceticus and Acinetobacter genomic species 3. J Clin Microbiol. 2010;48(12):4592-4.

16. Higgins PG, Wisplinghoff $H$, Krut $O$, Seifert $H$. A PCR-based method to differentiate between Acinetobacter baumannii and Acinetobacter genomic species 13TU. Clin Microbiol Infect. 2007;13(12):1199-201.

17. Woodford N, Ellington MJ, Coelho JM, Turton JF, Ward ME, Brown S, et al. Multiplex PCR for genes encoding prevalent OXA carbapenemases in Acinetobacter spp. Int J Antimicrob Agents. 2006;27(4):351-3.

18. Poirel L, Walsh TR, Cuvillier V, Nordmann P. Multiplex PCR for detection of acquired carbapenemase genes. Diagn Microbiol Infect Dis. 2011;70(1):119-23.

19. Afzal-Shah M, Woodford N, Livermore DM. Characterization of OXA-25, OXA-26, and OXA-27, molecular class D beta-lactamases associated with carbapenem resistance in clinical isolates of Acinetobacter baumannii. Antimicrob Agents Chemother. 2001;45(2):583-8.

20. Vila J, Marcos MA, Jimenez de Anta MT. A comparative study of different PCR-based DNA fingerprinting techniques for typing of the Acinetobacter calcoaceticus-A. baumannii complex. J Med Microbiol. 1996;44(6):482-9.

21. Bou G, Cervero G, Dominguez MA, Quereda C, Martinez-Beltran J. PCRbased DNA fingerprinting (rep-PCR, AP-PCR) and pulsed-field gel electrophoresis characterization of a nosocomial outbreak caused by imipenem- and meropenem-resistant Acinetobacter baumannii. Clin Microbiol Infect. 2000;6(12):635-43.

22. Diancourt L, Passet V, Nemec A, Dijkshoorn L, Brisse S. The population structure of Acinetobacter baumannii: expanding multiresistant clones from an ancestral susceptible genetic pool. PLoS One. 2010;5(4):e10034

23. Grundmann $H$, Hori S, Tanner G. Determining confidence intervals when measuring genetic diversity and the discriminatory abilities of typing methods for microorganisms. J Clin Microbiol. 2001;39(11):4190-2.

24. Kim UJ, Kim HK, An JH, Cho SK, Park KH, Jang HC. Update on the epidemiology, treatment, and outcomes of Carbapenem-resistant Acinetobacter infections. Chonnam Med J. 2014:50(2):37-44.

25. Wernli D, Jorgensen PS, Harbarth S, Carroll SP, Laxminarayan R, Levrat N, et al. Antimicrobial resistance: the complex challenge of measurement to inform policy and the public. PLoS Med. 2017;14(8):e1002378.

26. Mugnier PD, Poirel L, Naas T, Nordmann P. Worldwide dissemination of the bla $_{\mathrm{OXA}-23}$ carbapenemase gene of Acinetobacter baumannii. Emerg Infect Dis. 2010;16(1):35-40.

27. Wu W, He Y, Lu J, Lu Y, Wu J, Liu Y. Transition of bla OXA-58-like to bla OXA-23like in Acinetobacter baumannii clinical isolates in southern China: an 8-year study. PLoS One. 2015;10(9):e0137174

28. Lolans K, Rice TW, Munoz-Price LS, Quinn JP. Multicity outbreak of carbapenem-resistant Acinetobacter baumannii isolates producing the carbapenemase OXA-40. Antimicrob Agents Chemother. 2006;50(9):2941-5.

29. Nunez Quezada T, Rodriquez CH, Castro Canarte G, Nastro M, Balderrama Yarhui $\mathrm{N}$, et al. Outbreak of bla OxA-72-producing Acinetobacter baumannii in South America. J Chemother. 2017;29(5):321-4.

30. Sari AN, Bicmen M, Gulay Z. The first report on the outbreak of OXA-24/40like Carbapenemase-producing Acinetobacter baumannii in Turkey. Jpn J Infect Dis. 2013;66(5):439-42.

31. Merino M, Acosta J, Poza M, Sanz F, Beceiro A, Chaves F, et al. OXA-24 carbapenemase gene flanked by XerC/XerD-like recombination sites in different plasmids from different Acinetobacter species isolated during a nosocomial outbreak. Antimicrob Agents Chemother. 2010;54(6):2724-7.

32. Wang $H$, Guo $P$, Sun $H$, Wang $H$, Yang $Q$, Chen $M$, et al. Molecular epidemiology of clinical isolates of carbapenem-resistant Acinetobacter spp. from Chinese hospitals. Antimicrob Agents Chemother. 2007;51(11):4022-8.

33. Lemos EV, de la Hoz FP, Alvis N, Einarson TR, Quevedo E, Castaneda C, et al. Impact of carbapenem resistance on clinical and economic outcomes among patients with Acinetobacter baumannii infection in Colombia. Clin Microbiol Infect. 2014;20(2):174-80. 
34. Huang ST, Chiang MC, Kuo SC, Lee YT, Chiang TH, Yang SP, et al. Risk factors and clinical outcomes of patients with carbapenem-resistant Acinetobacter baumannii bacteremia. J Microbiol Immunol Infect. 2012;45(5):356-62.

35. Carlet J, Ben Ali A, Chalfine A. Epidemiology and control of antibiotic resistance in the intensive care unit. Curr Opin Infect Dis. 2004;17(4):309-16.

36. Chusri S, Silpapojakul K, McNeil E, Singkhamanan K, Chongsuvivatwong V. Impact of antibiotic exposure on occurrence of nosocomial carbapenemresistant Acinetobacter baumannii infection: a case control study. J Infect Chemother. 2015;21(2):90-5.

37. Surasarang K, Narksawat K, Danchaivijitr S, Siripanichgon K, Sujirarat D, Rongrungrueng $Y$, et al. Risk factors for multi-drug resistant Acinetobacter baumannii nosocomial infection. J Med Assoc Thail. 2007;90(8):1633-9.

38. Kim T, Chong YP, Park SY, Jeon MH, Choo EJ, Chung JW, et al. Risk factors for hospital-acquired pneumonia caused by carbapenem-resistant gramnegative bacteria in critically ill patients: a multicenter study in Korea. Diagn Microbiol Infect Dis. 2014;78(4):457-61.

39. Karah N, Sundsfjord A, Towner K, Samuelsen O. Insights into the global molecular epidemiology of carbapenem non-susceptible clones of Acinetobacter baumannii. Drug Resist Updat. 2012;15(4):237-47.

40. Ruan Z, Chen Y, Jiang Y, Zhou H, Zhou ZH, Fu Y, et al. Wide distribution of CC92 carbapenem-resistant and OXA-23-producing Acinetobacter baumannii in multiple provinces of China. Int J Antimicrob Agents. 2013;42(4):322-8.

41. Tacconelli E, Cataldo MA, Dancer SJ, De Angelis G, Falcone M, Frank U, et al. ESCMID guidelines for the management of the infection control measures to reduce transmission of multidrug-resistant gram-negative bacteria in hospitalized patients. Clin Microbiol Infect. 2014;20(Suppl 1):1-55.

42. Johnson JK, Robinson GL, Zhao L, Harris AD, Stine OC, Thom KA. Comparison of molecular typing methods for the analyses of Acinetobacter baumannii from ICU patients. Diagn Microbiol Infect Dis. 2016;86(4):345-50.

43. Thom KA, Hsiao WW, Harris AD, Stine OC, Rasko DA, Johnson JK. Patients with Acinetobacter baumannii bloodstream infections are colonized in the gastrointestinal tract with identical strains. Am J Infect Control. 2010;38(9):751-3.

44. Morris AK, Masterton RG. Antibiotic resistance surveillance: action for international studies. J Antimicrob Chemother. 2002;49(1):7-10.

Ready to submit your research? Choose BMC and benefit from:

- fast, convenient online submission

- thorough peer review by experienced researchers in your field

- rapid publication on acceptance

- support for research data, including large and complex data types

- gold Open Access which fosters wider collaboration and increased citations

- maximum visibility for your research: over $100 \mathrm{M}$ website views per year

At $\mathrm{BMC}$, research is always in progress.

Learn more biomedcentral.com/submissions 